

$340 \cdot 3$

\title{
los ruidos en las edificaciones $\mathbf{V}$
}

J. M. TOBIO, Dr. en Química Industrial

medidas acústicas

\section{0.-Necesidad de las medidas}

Para que las disposiciones a tomar en la defensa contra el ruido sean verdaderamente eficaces, es casi siempre necesario realizar medidas acústicas apropiadas.

En los últimos quince años se han experimentado grandes progresos en acústica $\mathrm{y}$, como consecuencia de ello, las medidas sónicas se han hecho más necesarias, más exactas y, al mismo tiempo, más sencillas.

Cuando se estudia un montaje nuevo-aislante, absorbente o anti-reverberante-, es casi imprescindible comprobar "in situ" los resultados previstos en el cálculo. Esto ha de hacerse, necesariamente, con ensayos y medidas acústicas. Las medidas son igualmente indispensables cuando se trata de obtener datos para proyectar un aislamiento o acondicionamiento fónicos.

En acústica aplicada pueden distinguirse dos clases de medidas: una las de sonidos o ruidos propiamente dichos, y otra las de las propiedades o características de los materiales o dispositivos de protección sonora. Las medidas de sonidos o ruidos que se tratan de disminuir o combatir se hacen directamente, como es obvio, en el sitio donde se producen. Las medidas de las propiedades de los materiales, son, en gran parte, medidas de laboratorio. Cuando se trata de estudiar el comportamiento de una disposición constructiva, es natural que los aparatos acústicos de laboratorio vayan a la obra misma.

Como ya se dijo en 3 , todas las medidas acústicas se reducen, en la práctica, a dos: intensidad y frecuencia. La intensidad se mide, casi exclusivamente, en decibelios; y la frecuencia, en ciclos por segundo (ver 1 y 2 ). 
En el apartado siguiente veremos que ambas magnitudes suelen medirse con el mismo aparato: el espectrómetro.

\section{1.-Medida de niveles sonoros y frecuencias}

Los aparatos mostrados en las figuras 4 y 6 son los más utilizados para la medida del nivel sonoro. El primero es un sencillo modelo de bolsillo (adecuado para llevar a cabo determinaciones iniciales de tanteo), calibrado en decibelios, pero que no permite discriminar sobre la frecuencia del sonido en estudio.

El analizador de octavas, o espectrómetro (fig. 6), es un aparato $\mathrm{mu}$ cho más completo, que sirve para obtener los "espectrogramas" del sonido. Está especialmente diseñado para hacer una exploración completa del campo audible y proporciona el nivel sonoro (en db) para cada frecuencia (en $\mathrm{c} / \mathrm{s}$ ).

Generalmente, este analizador se asocia con un registro gráfico, formando el conjunto representado en la figura 42 , en la cual se ve un micrófono a cristal (4.111), con pie (4.122), acoplado al analizador de octavas (2.109) que, a su vez, va conectado, mecánica y eléctricamente, al registro gráfico (2.304). Las curvas resultantes de esta disposición tienen un aspecto similar al indicado en la figura 43.

En esta figura se representan cuatro espectrogramas, que corresponden (de arriba a abajo) a los ruidos producidos por: un chorro de aire saliendo por un tubo, un motor eléctrico de una estampadora, un motor de una taladradora de mano, y el motor de una taladradora grande.
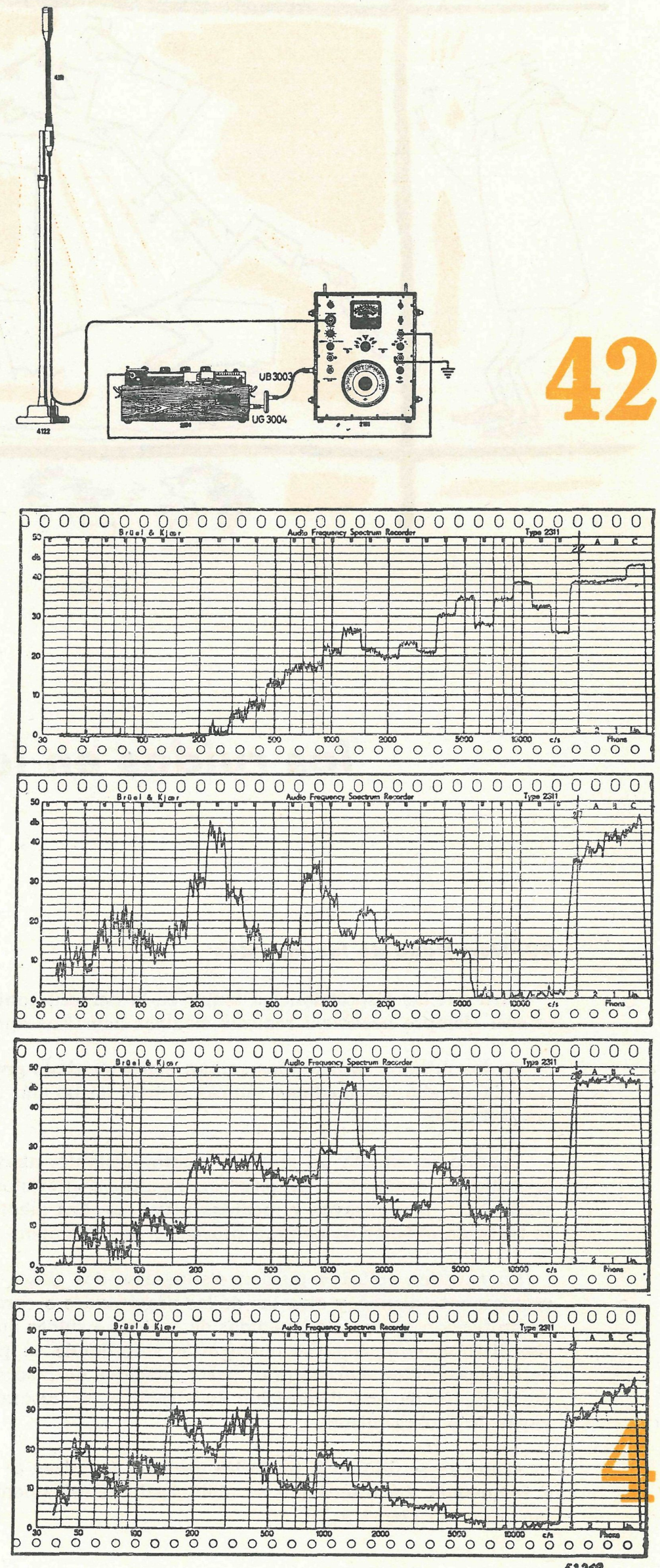

52919 
Los valores dados por las gráflcas de la figura 43 vienen dados en db (ordenadas) $8 \mathrm{c} / \mathrm{s}$ (abscisas). Son valores objetivos y representan, únicamente, la magnitud "física" del sonido, sin tener para nada en cuenta las caracteristicas fisiológicas del oído humano.

Si se tienen en cuenta estas características (principalmente la escasa sensibilidad del oído para las frecuencias bajas), será necesario traducir estos decibelios a fonios mediante las curvas de la figura 27. La unidad fonio ha sido ya definida en 2.

Esta unidad, aunque más representativa que el $\mathrm{db}$, tampoco tiene en cuenta otra característica del oído, cual es la pérdida de sensibilidad para los sonidos de elevada intensidad. Se ha establecido, por ello, una escala subjetiva de intensidades, que se mide en sones o sonios. Esta presenta la ventaja de que es "proporcional", es decir, un sonido de dos sonios es dos veces más intenso (subjetivamente) que uno de un sonio. A $1.000 \mathrm{c} / \mathrm{s}, 40 \mathrm{db}$ equivalen a un sonio.

El paso de fonios a sonios se hace fácilmente por medio de la gráflca de la figura 44 .

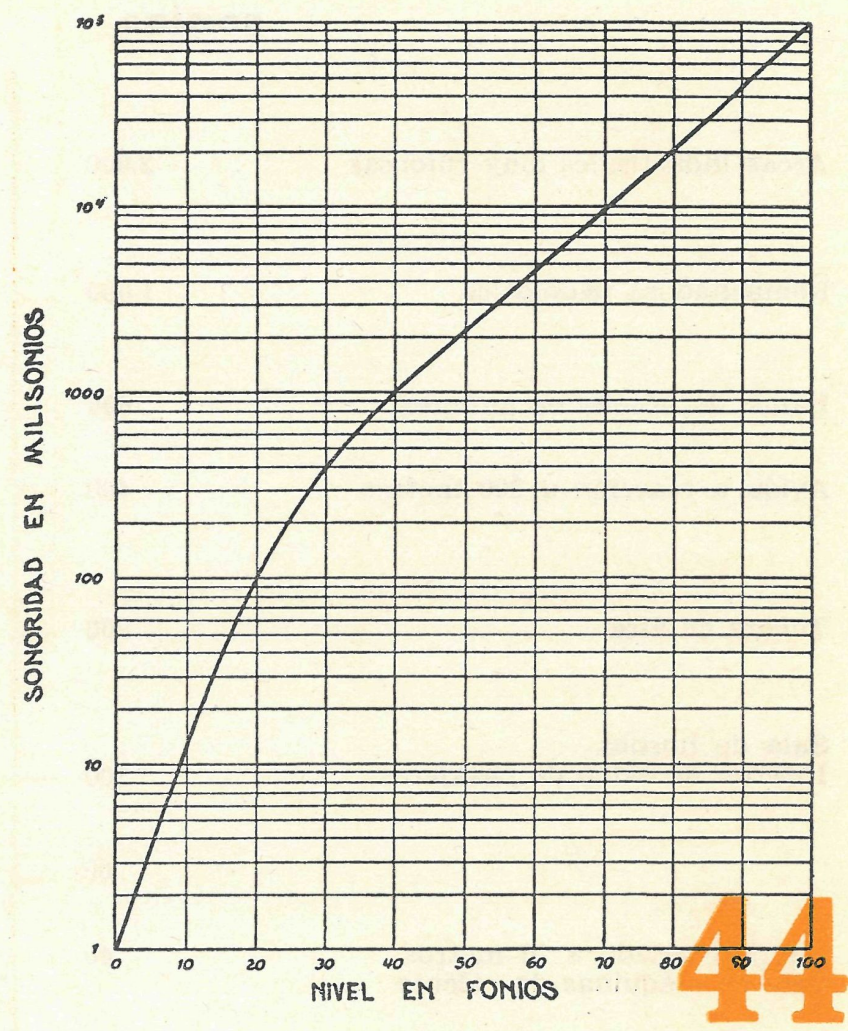

El paso de db a sonios puede hacerse utilizando sucesivamente las gráficas de las figuras 27 y 44. Pero se efectúa corrientemente con las tablas de paso db-sonios, incluídas en el Manual mencionado al final de este trabajo.

\section{2.-Sonoridad fotal}

La unidad son o sonio depende, por tanto, no sólo del nivel de presión sonora (intensidad), sino también de la frecuencia, e, incluso, de las características fisiológicas del oído. Es, consecuentemente, una buena magnitud para definir y medir la "sonoridad total", o sea, la sensación que un oyente experimenta según que los sonidos sean flojos o fuertes. Es decir, según la cantidad total de energía que es enviada al cerebro.

La sonoridad es una medida eminentemente subjetiva, que no puede realizarse con ningún aparato, y, para una presión sonora dada, puede ser mayor o menor según la frecuencia. En la práctica no es posible manejar las complicadas relaciones numéricas o gráficas que definen la sonoridad de un recinto. Es necesario dar una cifra que represente la sonoridad total y que pueda utilizarse tanto en los cálculos de aislamiento y acondicionamiento, como para fines de comparación.

Para ello existen diversos métodos. Uno de los más convenientes es el de Stevens. Consiste en hacer un espectrograma del sonido o ruido, que da directamente los niveles sonoros, en db, para las frecuencias standard $(100,125,160,200,250,320,400,500,640,800,1.000,1.250,1.600,2.000,2.500$, 3.200 y $4.000 \mathrm{c} / \mathrm{s}$ ).

Con estos valores en $\mathrm{db}$, se determinan, como se indicó anteriormente, los valores en sonios para cada frecuencia. Estos valores se suman aritméticamente (

Luego se aplica la fórmula,

$$
\mathbf{S}_{t}=\mathbf{S}_{m}+0,15\left(\mathbf{S} \mathbf{S}-\mathbf{S}_{m}\right)
$$

en la que $\mathbf{S}_{t}$ es la sonoridad total, en sonios, y $\mathbf{S}_{m}$ es el valor de la sonoridad más elevado encontrado, en sonios. 


\section{sonios}

Areas industriales muy ruidosas

Remachadora neumática

Banco de prueba de motores

Avión a reacción a 300 metros

Tobera de aire

Sala de hornos

Interior de avión de pasajeros

Camión pesado a 10 metros Sala de máquinas de cálculo

Interior de un automóvil

Limpiadora de vacío

Interior de un tren de viajeros

Avión comercial a 600 metros Radio y televisión

Oficina típica

Biblioteca pública

Apartamento de hotel

Oficina tranquila o estudio

Nevera

Sala de estar en hogar tranquilo

La más silenciosa área rural

Umbral aproximado de audición para ruidos de banda ancha

Umbral de audición para tonos puros

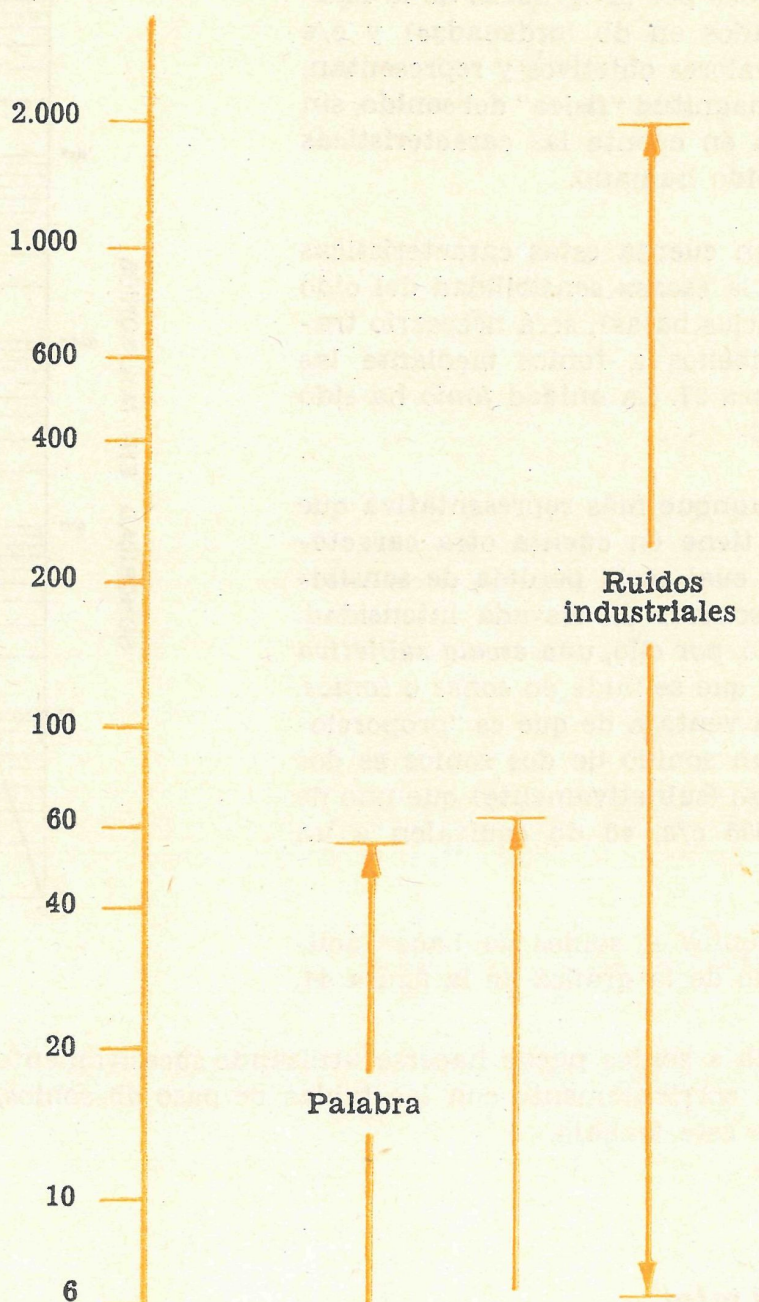

Ruidos de oficina
4

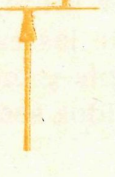

Ruidos del hogar$$
1
$$

0,6

0,4

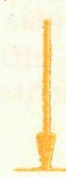

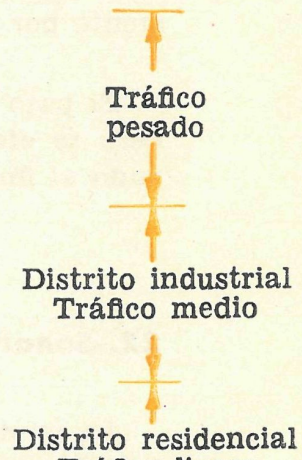

Tráfico ligero

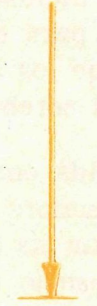

\section{sonoridad en diferentes ambientes o lugares de trabajo}




\section{3.-Sonoridad admisible}

Cuando ya se sabe determinar el valor de la sonoridad, puede preguntarse: ¿Cuánto ruido es aceptable en tal o cual circunstancia? ¿Cómo influye esta sonoridad sobre la conversación normal o telefónica?

En la figura 45 se indica una tabla o nomograma que muestra las sonoridades en determinados ambientes o lugares de trabajo. No hay que confundir este nomograma con el incluído en la figura 26. En el de la figura 45 se tiene en cuenta la "sonoridad total", calculada según 22 , y no los niveles acústicos en fonios (fig. 26).

En la figura 45 se muestran las sonoridades en zonas industriales y de oficinas, y para diversas máquinas. Como orientación para el constructor diremos que un sonio es, aproximadamente, el ruido producido por una nevera de compresor en una cocina. La sonoridad en locales de oficinas puede ir desde 0,5 a 40 sonios.

Por debajo de un sonio el ambiente suele considerarse como tranquilo; alrededor de 10 sonios es un ambiente algo ruidoso, y por encima de 50 sonios es intolerable para oficinas. Algunas máquinas ruidosas, utilizadas en ciertas oficinas, producen hasta 40 sonios.

Por encima de 30 sonios de sonoridad ambiente es prácticamente imposible llevar a cabo una conversación, salvo si se habla en la oreja del interlocutor. Con 10 sonios de ambiente es difícil hacerse entender por teléfono.

La práctica enseña que, para oficinas normales, pueden consentirse hasta 5-8 sonios, y para despachos tranquilos (dirección o estudio), unos dos sonios.
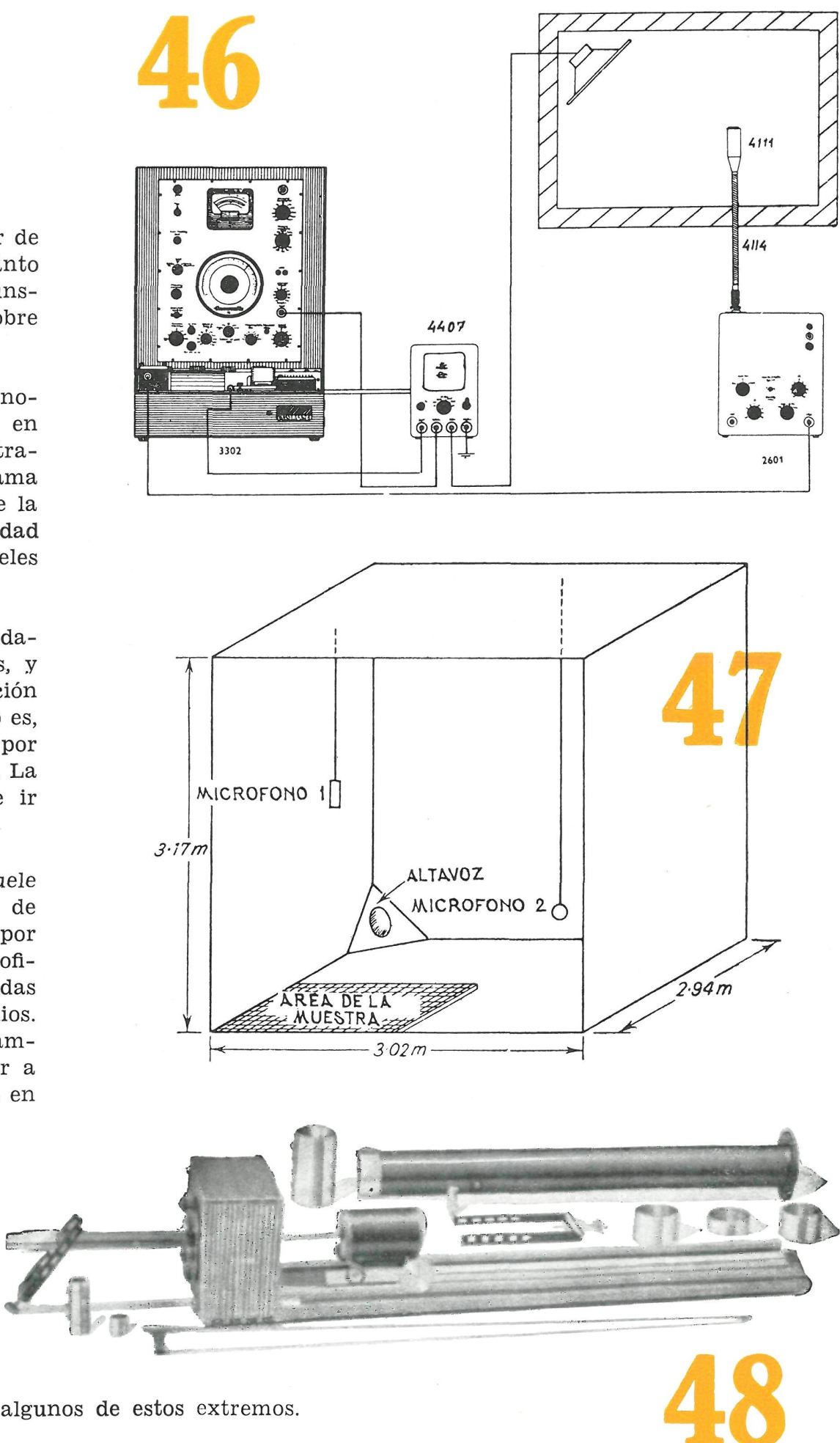

TABLA III

SONORIDAD EN OFICINAS

AMBIENTE

Extremadamente ruidoso Muy ruidoso

Ruidoso

Ligeramente ruidoso

Silencioso

Muy silencioso

\section{CONDICIONES PARA LA CONVERSACION}

Prácticamente imposible Difícil

Fácil
RECOMENDADO PARA...

Oficina ruidosa, con máquinas

Oficina normal

Oficina tranquila 


\section{4.-Medida del tiempo de reverberación}

En 4 ya se explicó la importancia del tiempo de reverberación de una habitación o recinto, y en la figura 9 se muestra el esquema experimental más utilizado para su medida. Esta disposición es válida para la medida a una sola frecuencia (generalmente, $1.250 \mathrm{c} / \mathrm{s}$ ).

Cuando se quiere obtener el valor $t_{60}$ para un espectro completo de frecuencias se adopta la disposición mostrada en la figura 46. En ella existe un oscilador de baja frecuencia (análogo al indicado en la figura 9), acoplado, mecánica y eléctricamente, a un registro gráfico (3.202, en la figura 46), y éste, a su vez, unido a un conmutador automático (4.407), encargado de conectar y desconectar el altavoz que, con el micrófono (4.111), se encuentra dentro del recinto en estudio. Existe también el amplificador de micrófono (2.601), análogo al empleado en la figura 9.

Si no se dispone de aparato alguno, pero se conocen el volumen del recinto y la calidad acústica absorbente de los materiales de que está interiormente recubierta la habitación, es fácil calcular el tiempo de reverberación "teórico" mediante la fórmula [7]. El valor obtenido puede servir como orientación.

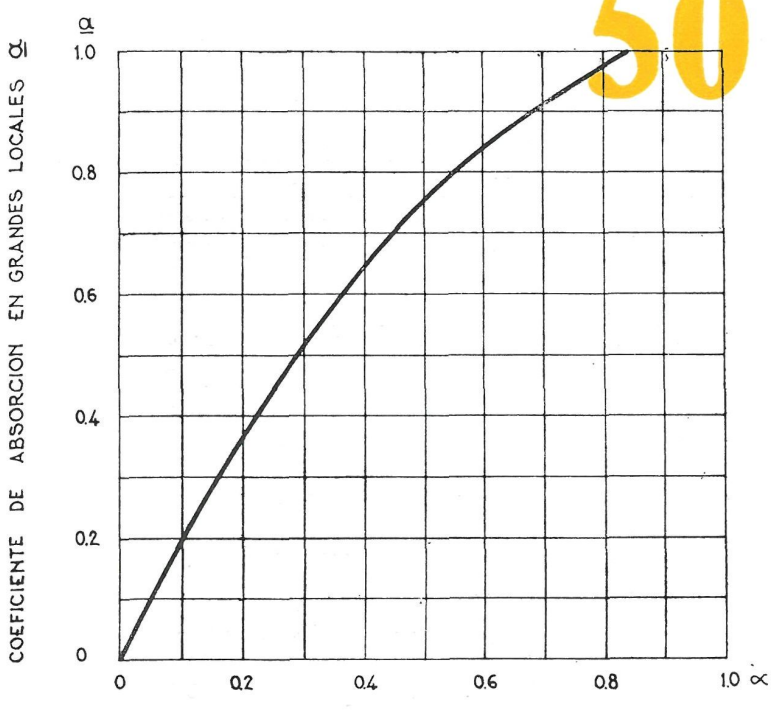

COEFICIENTE DE A'BSORCIOH: $\propto$ POR EL METODO DEL TUBO
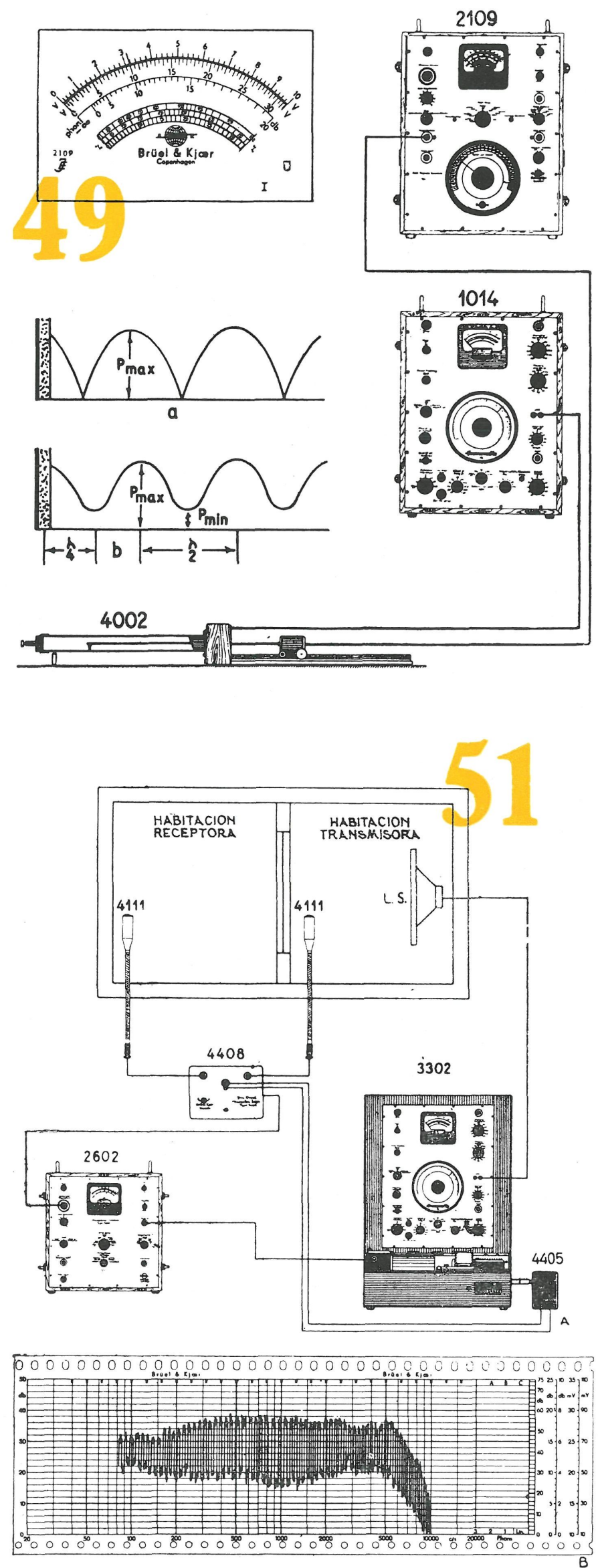


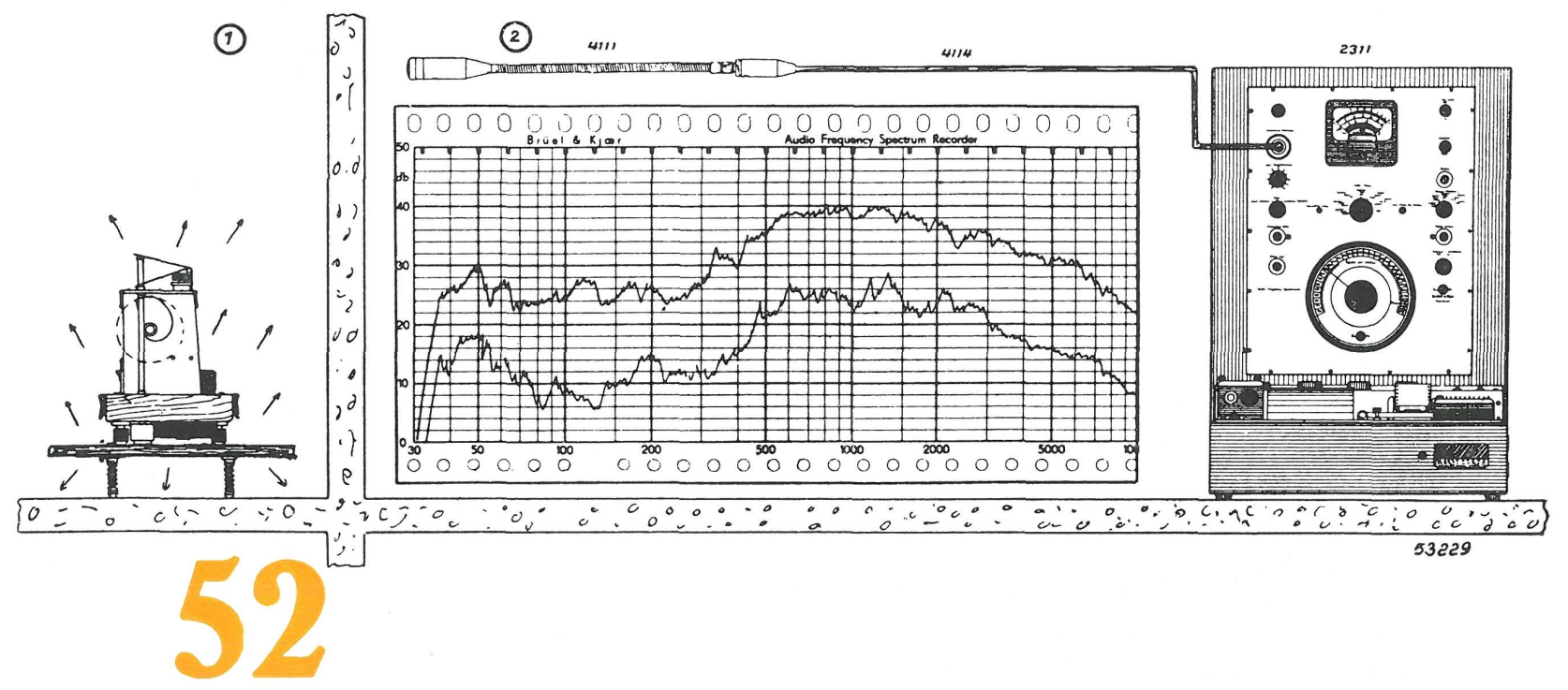

\section{7.-Laboratorios móviles}

Muchos de los ensayos acústicos descritos han de realizarse "in situ", lo cual obliga a desplazar a la obra costosos y pesados aparatos. El acarreo, montaje y recogida de estos aparatos supone, casi siempre, una inversión de tiempo mucho mayor que el ensayo mismo.

Por ello se han proyectado remolques o furgonetas especialmente acondicionados, para que estos aparatos vayan debidamente montados, instalados y conectados. De este modo, los únicos accesorios que deben llevarse al interior de la obra son los micrófonos y los productores de ruidos (altavoz o aparato de martillos), lo cual supone una gran comodidad.
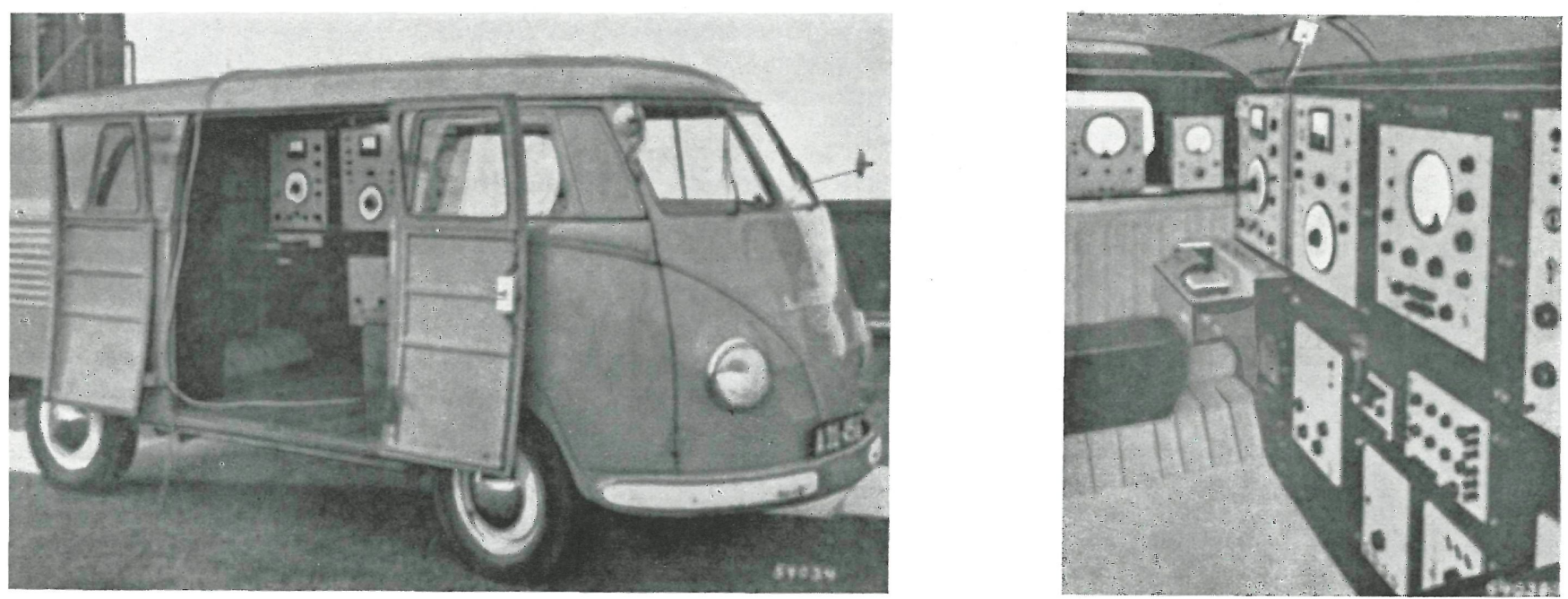


\section{5.-Medida del coeficiente de absorción}

El coeficiente $\alpha$ (ver 4, 6, 7 y 8) para un material absorbente puede determinarse de dos modos: en cámaras reverberantes y por el método de las ondas estacionarias.

La mencionada fórmula [7] permite, en combinación con una cámara reverberante, determinar el coeficiente de absorción de paneles o láminas de material absorbente. La cámara reverberante suele ser de forma más o menos cúbica, de paredes gruesas, duras y lisas interiormente. Sobre la cámara desnuda se determina el tiempo de reverberación $t_{60}$.

Si $\mathrm{S}_{o}$ es la superficie interior total de la cámara, $\mathrm{y} \alpha_{o}$ el coeficiente de absorción de las paredes desnudas, y $\mathrm{V}$ el volumen de la misma, la aplicación de la fórmula [7] permite obtener:

$$
\alpha=\frac{\alpha_{o}}{\mathrm{~S}}\left[\mathrm{~S}_{0} \frac{t_{60}}{t_{60}^{\prime}}+\mathrm{S}-\mathrm{S}_{0}\right]
$$

Una disposición experimental para el ensayo nos la muestra la figura 47. En lugar del altavoz en el vértice pueden emplearse dos altavoces separados, de tal modo que se logre una correcta distribución del sonido.

Otra forma de determinar $\alpha$ es por medio del aparato de ondas estacionarias, cuyos componentes se ven agrupados en la figura 48. La caja rectangular contiene un altavoz que envía su energía sonora por el interior de un tubo cilíndrico (hay dos, de diámetros diferentes) cerrado por un extremo. En este extremo se coloca una probeta cilíndrica, de espesor máximo de 95 milímetros.

Un micrófono, contenido en el recipiente cilíndrico con ruedas, visible en la figura 48, va provisto de una varilla-sonda que explora el campo de ondas estacionarias creado en el intèrior del tubo, detectando los puntos de máxima y mínima intensidad.

Como aparatos complementarios, se emplean (fig. 49) un oscilador calibrado (1.014) y un espectrómetro (2.109). Este último lleva una escala (I) calibrada directamente en valores de $\alpha$.

Los valores de $\alpha$ hallados con pequeñas probetas en el aparato de ondas estacionarias son, en general, inferiores a los encontrados en láminas con las cámaras reverberantes. El paso de uno a otro valor puede hacerse con la curva de la figura 50. Para los cálculos de absorción debe utilizarse, preferentemente, el valor dado para las cámaras.

\section{Medida de aislamientos}

La determinación del poder aislante acústico de un material puede hacerse en el laboratorio o en obra. En el primer caso se requieren dos cámaras reverberantes con un orificio de comunicación entre ambas, de $2,5 \times 2,5 \mathrm{~m}$, aproximadamente. Tapando esta abertura se coloca la placa, elemento de tabique o muro, ventana, puerta o cualquier panel sólido cuyo poder aislante (pérdidas de transmisión, 5) se quiere determinar.

Una de las cámaras (figura 51), que es la transmisora, lleva un altavoz (L. S.) y un micrófono (4.111). En la cámara receptora hay otro micrófono (4.111). Un oscilador de baja frecuencia (3.302), conectado a un registro gráfico, envía la corriente de audiofrecuencia al altavoz. El nivel sonoro, antes y después del panel en ensayo colocado entre ambas cámaras, se mide con los micrófonos conectados a un conmutador (4.408), que conecta alternativamente uno u otro. La señal es amplificada por (2.602) antes de ser enviada al registro.

Sobre este último aparecen dos gráficas (parte inferior de la figura 51), que representan los niveles en las dos cámaras. La diferencia de ordenadas en estas gráficas da directamente, en db, las pérdidas de transmisión para cada frecuencia.

Cuando se pretende determinar el aislamiento de un tabique, muro o forjado, en obra, suele emplearse un aparato productor de ruido de cinco martillos (aunque también puede utilizarse un altavoz), colocado a un lado del muro en cuestión (fig. 52). La técnica de las medidas es similar a la explicada en los párrafos anteriores.

En el esquema representado (fig. 52) se emplea un solo micrófono (4.111), conectado a un espectrómetro (2.311) provisto de su correspondiente registro gráfico. Se hacen dos lecturas, una con el micrófono en la habitación (1) (curva superior), y otra con el micrófono en la habitación (2) (curva inferior). La diferencia de ordenadas, en cada frecuencia, da directamente las pérdidas de transmisión en db. 
En general, un laboratorio de este tipo debe ir equipado con los siguientes aparatos: oscilador de audiofrecuencia, espectrómetro de audio, registro gráflco, amplificadores de micrófono, varios micrófonos, pies para los mismos, uno o dos altavoces, aparato normalizado de martillos, selector automático para conexión a dos micrófonos, cables de conexión y pequeño material eléctrico.

Las figuras 53 y 54 muestran algunos detalles de cómo pueden disponerse los diversos aparatos acústicos en una furgoneta-laboratorio.

El Instituto Técnico de la Construcción y del Cemento dispone de uno de estos laboratorios, debidamente equipado, con el cual ya se han realizado diversas medidas acústicas, tanto en Madrid como en provincias.

Nora: Una explicación más detallada sobre algunos de los puntos tratados en esta serie de artículos puede verse en el manual de este Instituto: "El aislamiento y acondicionamiento acústico en la edificación" (en prensa). También se incluyen en él una serie de datos prácticos y tablas referentes a problemas de defensa contra el ruido.

\section{Apéndices}

\section{TABLA 1}

"Niveles sonoros, en db, en función de la frecuencia"

FUENTE DE RUIDO A $6 \mathrm{~m}$

Avión de reacción de cuatro motores

Gran orquesta sinfónica

Ferrocarril eléctrico

Automóvil, a $80 \mathrm{~km} / \mathrm{hr}$, sobre autopista de hormigón

Soplante de $10 \mathrm{HP}$

Voz humana, gritando

Voz humana, conversación normal

Ruido de máquinas de escribir

Sala de estar, tranquila

\begin{tabular}{rrrrrr}
\multicolumn{7}{c}{ CICLOS POR SEGUNDO } \\
125 & 250 & $\mathbf{5 0 0}$ & 1.000 & 2.000 & 4.000 \\
132 & 135 & 136 & 132 & 129 & 125 \\
80 & 85 & 86 & 83 & 82 & 80 \\
84 & 81 & 80 & 78 & 72 & 65
\end{tabular}

$\begin{array}{llllll}85 & 77 & 76 & 73 & 70 & 72\end{array}$

$\begin{array}{llllll}69 & 72 & 72 & 72 & 67 & 65\end{array}$

$\begin{array}{llllll}60 & 64 & 67 & 68 & 63 & 55\end{array}$

$\begin{array}{llllll}48 & 51 & 56 & 57 & 51 & 43\end{array}$

$\begin{array}{llllll}40 & 44 & 48 & 49 & 51 & 53\end{array}$

$\begin{array}{llllll}45 & 53 & 38 & 33 & 21 & 20\end{array}$

TABLA 2

"Coeficiente de absorción de algunos materiales"

\begin{tabular}{|c|c|c|c|c|c|c|}
\hline \multirow{2}{*}{ M A TERIAL } & & \multicolumn{4}{|c|}{ GICLOS POR SEGUNDO } & \multirow{2}{*}{4.000} \\
\hline & 125 & 250 & $\mathbf{5 0 0}$ & 1.000 & 2.000 & \\
\hline Hormigón desnudo & 0,01 & 0,01 & 0,02 & 0,02 & 0,02 & 0,03 \\
\hline Hormigón pintado & 0,01 & 0,01 & 0,01 & 0,02 & 0,02 & 0,02 \\
\hline Ladrillo visto & 0,024 & 0,025 & 0,031 & 0,04 & 0,05 & 0,07 \\
\hline Ladrillo pintado & 0,012 & 0,013 & 0,017 & 0,02 & 0,023 & 0,025 \\
\hline Piedra (igual que hormigón) & - & - & - & - & - & - \\
\hline Bloques de hormigón & 0,3 & 0,45 & 0,3 & 0,25 & 0,4 & 0,25 \\
\hline Contrachapado madera & 0,11 & - & 0,12 & - & 0,1 & - \\
\hline Enlucido yeso liso & 0,024 & 0,027 & 0,03 & 0,037 & 0,019 & 0,034 \\
\hline Enlucido mortero arena & 0,04 & 0,05 & 0,06 & 0,08 & 0,04 & 0,06 \\
\hline Enlucido rugoso & 0,025 & 0,026 & 0,06 & 0,085 & 0,043 & 0,056 \\
\hline Muro de ladrillo con enlucido de yeso & 0,013 & 0,015 & 0,02 & 0,028 & 0,04 & 0,05 \\
\hline Suelo de goma & 0,04 & 0,04 & 0,07 & 0,11 & 0,03 & 0,01 \\
\hline Estera flbra vegetal & 0,08 & 一 & 0,17 & - & 0,3 & - \\
\hline Aglomerado de corcho de $25 \mathrm{~mm}$ & - & 0,08 & 0,3 & 0,31 & 0,28 & - \\
\hline
\end{tabular}


TABLA 3

"Coeficiente de absorción de materiales absorbentes"

\begin{tabular}{|c|c|c|c|c|c|c|}
\hline \multirow{2}{*}{ M A TER I AL } & \multicolumn{6}{|c|}{ CICLOS POR SEGUNDO } \\
\hline & 125 & 250 & 500 & 1.000 & 2.000 & 4.000 \\
\hline $\begin{array}{l}\text { "Corkoustic" Armstrong, } 25,4 \mathrm{~mm} \text {, pegado al en- } \\
\text { lucido o forjado }\end{array}$ & 0,03 & 0,12 & 0,52 & 0,62 & 0,41 & 0,46 \\
\hline $\begin{array}{l}\text { "Corkoustic" Armstrong, } 25,4 \mathrm{~mm} \text {, suspendido me- } \\
\text { cánicamente }\end{array}$ & 0,11 & 0,47 & 0,50 & 0,27 & 0,44 & 0,50 \\
\hline $\begin{array}{l}\text { "Cushiontone" Armstrong, } 25,4 \mathrm{~mm} \text {, pegado al } \\
\text { techo }\end{array}$ & 0,18 & 0,39 & 0,89 & 0,97 & 0,77 & 0,60 \\
\hline $\begin{array}{l}\text { "Cushiontone" Armstrong, } 25,4 \mathrm{~mm} \text {, suspendido } \\
\text { mecánicamente }\end{array}$ & 0,41 & 0,56 & 0,78 & 0,91 & 0,80 & 0,64 \\
\hline "Vitrofib", $40 \mathrm{~mm}$ espesor & 0,20 & 0,35 & 0,65 & 0,80 & 0,75 & 0,65 \\
\hline "Vitroflb", $50 \mathrm{~mm}$ espesor & 0,35 & 0,60 & 0,77 & 0,85 & 0,83 & 0,75 \\
\hline "Vitrofib", $100 \mathrm{~mm}$ espesor & 0,75 & 0,95 & 0,97 & 0,92 & 0,85 & 0,78 \\
\hline "Asbestospray", $10 \mathrm{~mm}$ grueso & 0,28 & 0,23 & 0,64 & 0,78 & 0,87 & 0,64 \\
\hline "Asbestospray", $15 \mathrm{~mm}$ grueso & 0,31 & 0,26 & 0,81 & 0,88 & 0,90 & 0,91 \\
\hline "Asbestospray", $20 \mathrm{~mm}$ grueso & 0,35 & 0,31 & 0,85 & 0,91 & 0,89 & 0,01 \\
\hline "Asbestospray", $25 \mathrm{~mm}$ grueso & 0,44 & 0,53 & 0,86 & 0,92 & 0,95 & 0,98 \\
\hline
\end{tabular}

Nota: La mayor parte de los materiales incluídos en la Tabla 3 se encuentran en el mercado nacional.

TABLA 4

"Pérdidas de transmisión (en db) de algunos materialeṣ"

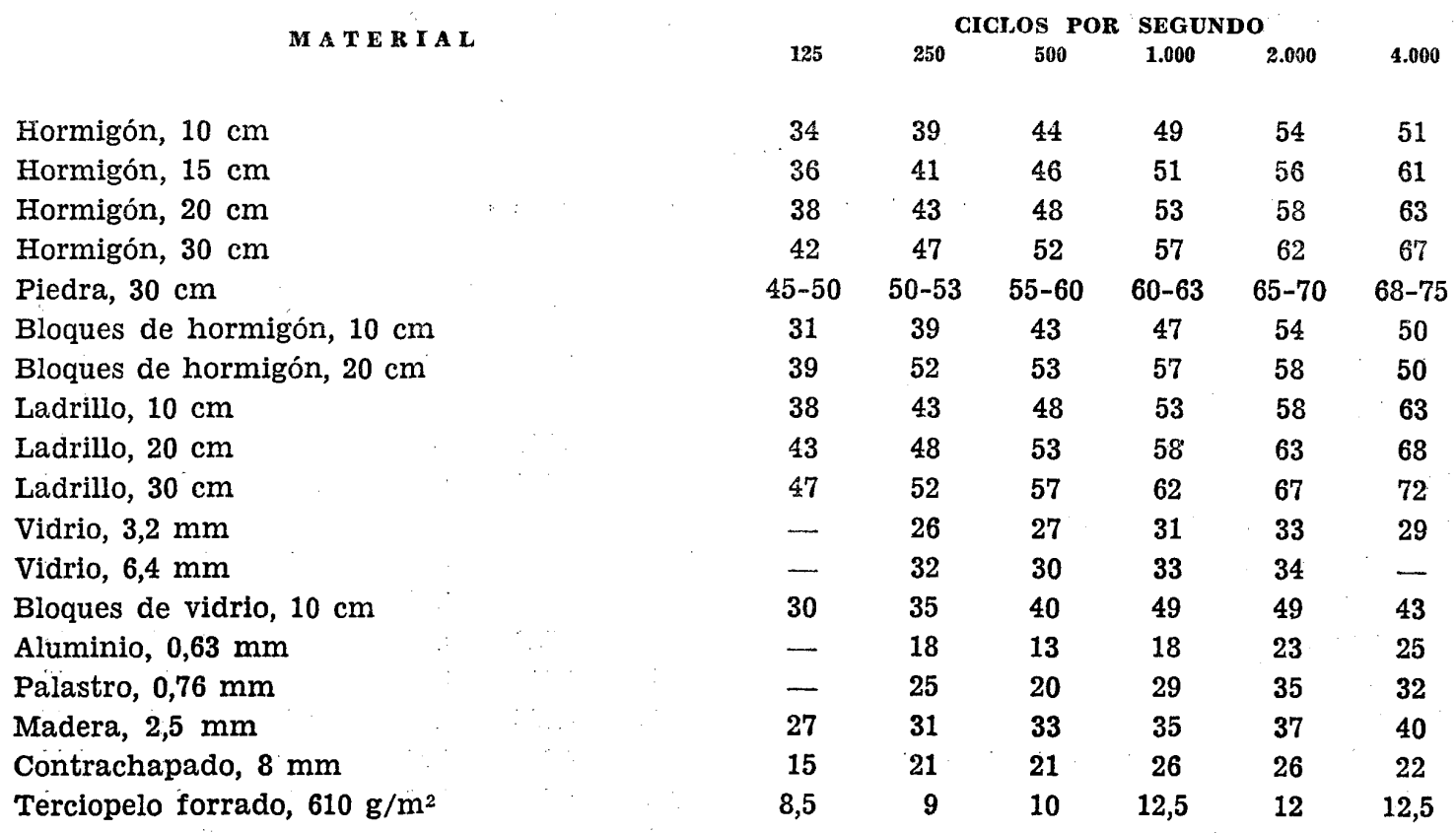

Nota: Ver también Tabla II, INFORMES DE LA Construccrón, núm. 109, art. 340-3. 